\title{
Decision of Multimodal Transportation Scheme Based on Swarm Intelligence
}

\author{
Kai Lei, ${ }^{1}$ Xiaoning Zhu, ${ }^{1}$ Jianfei Hou, ${ }^{2}$ and Wencheng Huang ${ }^{1}$ \\ ${ }^{1}$ School of Traffic \& Transportation, Beijing Jiaotong University, Beijing 100044, China \\ ${ }^{2}$ China Academy of Transportation Science, Beijing 100029, China \\ Correspondence should be addressed to Xiaoning Zhu; leikai@bjtu.edu.cn
}

Received 10 January 2014; Revised 20 March 2014; Accepted 20 March 2014; Published 24 April 2014

Academic Editor: Rui Mu

Copyright (c) 2014 Kai Lei et al. This is an open access article distributed under the Creative Commons Attribution License, which permits unrestricted use, distribution, and reproduction in any medium, provided the original work is properly cited.

\begin{abstract}
In this paper, some basic concepts of multimodal transportation and swarm intelligence were described and reviewed and analyzed related literatures of multimodal transportation scheme decision and swarm intelligence methods application areas. Then, this paper established a multimodal transportation scheme decision optimization mathematical model based on transportation costs, transportation time, and transportation risks, explained relevant parameters and the constraints of the model in detail, and used the weight coefficient to transform the multiobjective optimization problems into a single objective optimization transportation scheme decision problem. Then, this paper is proposed by combining particle swarm optimization algorithm and ant colony algorithm (PSACO) to solve the combinatorial optimization problem of multimodal transportation scheme decision for the first time; this algorithm effectively combines the advantages of particle swarm optimization algorithm and ant colony algorithm. The solution shows that the PSACO algorithm has two algorithms' advantages and makes up their own problems; PSACO algorithm is better than ant colony algorithm in time efficiency and its accuracy is better than that of the particle swarm optimization algorithm, which is proved to be an effective heuristic algorithm to solve the problem about multimodal transportation scheme decision, and it can provide economical, reasonable, and safe transportation plan reference for the transportation decision makers.
\end{abstract}

\section{Introduction}

\subsection{Basic Concepts}

(1) Swarm Intelligence. Swarm intelligence refers to the overall intelligent behavior emerging from interaction of many simple acts of individual; the swarm intelligence can produce a number of different individual collective behaviors. Swarm intelligence is the creative application and development of the collective behaviors and forms a more advanced whole wisdom. Swarm intelligence is a hot research topic in artificial intelligence researches in recent years, with no centralized control, does not provide a global model, and provides a complex distributed solution.

(2) Multimodal Transportation. Multimodal transportation is defined as completing the transportation process by using at least two transportation tools to connect and transport together; it is a kind of transportation organization form which uses optimum efficiency as the goal. There are no new transportation channels and tools in the multimodal transportation process; it is a combination of modern organization means and single transportation mode. It has important and realistic meaning for saving transportation costs and transportation time and improving transportation service quality by researching the decision of multimodal transportation scheme in the process of transportation.

\subsection{The Current Analysis Situation of the Domestic and Foreign Research}

(1) Swarm Intelligence. In recent years, the scholars at home and abroad carried out a lot of research works about swarm intelligence; the swarm intelligence theory in transportation related fields has been applied extensively. Chen and $\mathrm{Ma}$ 
[1] gave a new algorithm of glowworm swarm optimization algorithm which was based on swarm intelligence optimization principle and successfully applied it to the 0-1 knapsack problem; Li et al. [2] proposed the Harvard structure and swarm intelligence optimization system based on the CTLS model, which used swarm intelligence based on calculation and source of agent in agent technology and the simulation based optimization theory, and the feasibility and reliability of the modeling optimization method are verified by simulation analysis of CTLS half bridge scheduling problem; Mi [3] proposed an optimization algorithm for execution efficiency and convergence problems of integrated design process using genetic algorithm for task scheduling; the genetic algorithm was improved and combined with the particle swarm algorithm by considering the effect of coupling relationship between tasks to the task execution results. It is verified with an example; the results showed that the algorithm has a fast convergence speed and stable result; $\mathrm{Li}$ et al. [4] established automated warehouse single pock horizontally rotating storage shelf system mathematical model and used the ant colony optimization algorithm to solve the path planning problem based on the goods selection. This method is capable of rotating storage rack system for rapid selection of goods and finds the optimal goods picking path in the global, high quality solution and short calculation time; $\mathrm{Wu}$ and Shi [5] established ant colony algorithm based on the improved meeting algorithm and proposed ant colony algorithm ant touring quality, meeting algorithm, and a parallel algorithm which is combined with the proposed strategy; the experimental results show that this algorithm has better effectiveness; Xiao et al. [6] proposed the mathematical model of concurrent tolerance optimization design and then mapped into a special traveling salesman problem order multiple traveling salesman problem; so as to reduce the difficulty of problem solving, they proposed a hybrid swarm intelligence algorithm for concurrent tolerance optimization design problem. Through calculation examples, the efficiency would be hybrid swarm intelligence algorithm with genetic algorithm and simulated annealing algorithm has better search ability and higher. Ma et al. [7] minimized the energy consumption and delay time, improved the evolutionary algorithms for solving the traveling salesman problem based on artificial neural network algorithm, and established the swarm intelligence analysis model and used it to analyze the lines planning for public travel. The experimental results showed that, the improved hybrid intelligent computing method was simple and effective and helped to overcome the blindness of algorithm selection, to further expand the research direction of computational intelligence. From the above research literatures, it is not difficult to see that the swarm intelligence has not only attracted much attention in recent years but also has a lot of space in the field of transportation applications. This paper was proposed by combining particle swarm optimization algorithm and ant colony algorithm (PSACO) to solve the combinatorial optimization problem of multimodal transportation scheme decision for the first time which can make up shortcomings of their own at the same time.
(2) Decision of Multimodal Transportation Scheme. In recent years, scholars at home and abroad did a lot of research works on how to choose the proper transportation mode in the multimodal transportation access. Among them, Lozano and Storchi's [8] research was about the problem of shortest feasible path in the multimodal transportation and used the sequential algorithm to solve the problem; Calvete et al. [9] studied the VRP with soft time window vehicles path problem and used the goal programming method to solve the problem. Li and Zhao [10] used the minimum total cost as the optimization objective, which included transportation cost, transfer cost and penalty cost; the goods delivery time and transportation capacity were used as constraint conditions; they built mixed integer linear programming model and designed the integer coding genetic algorithm. Wang et al. [11] studied and established the multimodal transportation 01 integer programming model under the time and capacity constraints, designed the natural number coding genetic algorithm based on the characteristics of the model, and finally tested validity of the model and the algorithm with examples. Feng and Zhang [12] considered the environmental pollution, energy consumption, and safety factors into multimodal transportation sustainable development and then introduced the concept of total society cost of multimodal transportation, used this minimum cost value as a whole object, and built a multimodal transportation collaborative optimization model. Tong and Nie [13] transformed intermodal path optimization problem into a generalized shortest path problem, used time and cost as optimization objective, established multimodal transportation mathematical model for the optimization of path changes in volume, and solved and verified actual problem by using ant colony algorithm. Zhang [14] deeply studied the whole container multimodal transportation network, expounded the key elements and the operation mechanism of multimodal container transportation network in the whole system, and, in accordance with the existing problems, put forward the basic countermeasures rationalization. Li and Zeng [15] improved path shortest time model under the multimodal transportation in the fourth party logistics, gave the transportation cost model based on satisfactory time path under the condition of multimodal transportation, and improved genetic algorithm to solve the problem. Wen [16] used multimodal container transportation optimization as the research object and discussed the optimization method of container multimodal transportation routes, transportation, and time constraints modes. Li [17] discussed the development of transportation mode choice as well as the main transportation mode selection method and then put forward the concept of generalized transportation cost. Wang [18], in order to achieve time delivery, reduced the cost, according to the actual situation of the key problem of logistics distribution in the process of transportation and traffic network of multimodal transportation with time window of minimum cost problem. Zhang [19] analyzed Chinese railway container transportation features and operation process, used the transportation path selection as the goal to improve the efficiency of container transport and shorten the container transport cycle, and reduced transportation costs for the purpose; then he used the optimization theory and 
operations research theory to establish the railway container transportation route choice model.

There are some shortcomings existing in the domestic and foreign documents all above about the multimodal transportation modes selection; the main problem is that the impact of transportation cost and time selection on multimodal transportation modes was considered in the past; only a single target situation for multimodal transportation mode optimal selection failed to consider other factors in the problems. But, in the actual decision process selection in multimodal transportation modes, in order to ensure the continuity and safety, we need to consider transportation safety and transportation risk factors comprehensively; only in this way we can meet the actual needs of transportation.

Multimodal transportation is a kind of holistic and integrated operation process about the mutual coordination and cooperation between multimodal transportation network nodes, node enterprises lines, social economic conditions, and natural environment factors. Risk factors existed in several aspects of the multimodal transportation network, including multimodal transportation enterprises, transportation channels, external economic, social environment, and natural factors; these risk factors are not only the necessary conditions for the formation of multimodal transportation network risk but also an important prerequisite for the existence of multimodal transportation network risk.

The transportation risk is one of the important factors of the multimodal transportation selection in this paper, but it can be found from the domestic and foreign documents' researches that few scholars considered transportation risk as the important factor, when they conducted researches in the choice of transportation mode and multimodal transportation problems; so the present researches cannot be well adapted to the actual situation of multimodal transportation modes selection; it is also difficult to give correct guidance to practice and reference.

\section{Establishment of the Optimization Model}

2.1. Problem Description. We suppose that a company has a batch of goods to transport from origin point $\mathrm{O}$ to destination point $\mathrm{D}$ through a multimodal transportation network; a plurality of hub nodes composed the multimodal transportation network; several transportation modes can be chosen between two arbitrary adjacent nodes; each node is a transit hub for different transportation modes; when we switched the goods from one mode to another mode, we need to spend some time and cost, but total transportation time cannot exceed the scope of delivery time. We hoped to deliver the goods safely, economically, and conveniently through the proper choice of the transportation modes during the agreed period.

\subsection{Making the Assumption}

(1) We have known and fixed all the relevant constants;

(2) there is one transportation mode existent at least on any section of known delivery path;
(3) the same batch of goods cannot be separated during the transport process; that is to say, we can only choose one transportation mode in the known arbitrary section path when delivering one batch of goods;

(4) the goods are transited at hub nodes, and the transit for each batch of goods in each hub node occurs one time at most.

(5) when choosing the transportation model, just consider the influence of the transportation operation risk, not including the others.

\subsection{Description of the Model Parameters}

$C_{i, i+1}^{k}$ is the per unit cost of the selected $k$ transportation mode from the node $i$ to the node $i+1$.

$d_{i}^{k, l}$ is the unit transfer cost from the transportation mode $k$ to transportation mode $l$ in the node $i$.

$t_{i, i+1}^{k}$ is transportation time of the selected $k$ transportation mode from the node $i$ to the node $i+1$.

$f_{i}^{k, l}$ is transfer time from the transportation mode $k$ to transportation mode $l$ in the node $i$.

$R T$ is the risk factors of the delivery goods.

$R C_{i, i+1}^{k}$ is the transportation risk factor of the selected $k$ transportation mode from the node $i$ to the node $i+1$.

$R F_{i}^{k, l}$ is the transfer risk factor from the transportation mode $k$ to transportation mode $l$ in the node $i$.

$A_{i, i+1}^{k}$ is the transportation ability of the selected $k$ transportation mode from the node $i$ to the node $i+1$. Consider

$x_{i, i+1}^{k}= \begin{cases}1, & \text { select } k \text { transportation mode from } \\ & \text { the node } i \text { to the node } i+1 \\ 0, & \text { otherwise, }\end{cases}$

$y_{i}^{k, l}= \begin{cases}1, & \text { from the transportation mode } \\ k \text { to transportation mode } l \text { in the node } i \\ 0, & \text { otherwise. }\end{cases}$

$T$ is the actual goods delivery time, $w$ is the earliest goods delivery time, and $v$ is the latest goods delivery time, $v \geq T \geq w>0$.

$I_{a}$ is each unit goods warehousing cost when delivery advanced and $V_{d}$ is each unit goods penalty cost when delivery is late. 
$J_{a}$ is all goods warehousing cost when delivery advanced; $J_{a}=\max \left[0,(w-T) I_{a}\right]$.

$S_{d}$ is all goods penalty cost when delivery is late; $S_{d}=$ $\max \left[0,(T-v) V_{d}\right]$;

$M$ is a sufficiently large penalty factor.

\subsection{Establishment of the Model}

(1) Objective 1: the least transportation cost for the decision of multimodal transportation scheme is as follows:

$$
\begin{aligned}
\operatorname{Min} Z= & \sum_{i=1}^{n} \sum_{k=1}^{m} C_{i, i+1}^{k} X_{i, i+1}^{k}+\sum_{i=1}^{n} \sum_{k=1}^{m} \sum_{l=1}^{m} d_{i}^{k, l} y_{i}^{k, l} \\
& +\max \left[0,(w-T) I_{a}\right]+\max \left[0,(T-v) V_{d}\right]
\end{aligned}
$$

(2) Objective 2: the least transportation time for the decision of multimodal transportation scheme is as follows:

$$
\operatorname{Min} T=\sum_{i=1}^{n} \sum_{k=1}^{m} t_{i, i+1}^{k} x_{i, i+1}^{k}+\sum_{i=1}^{n} \sum_{k=1}^{m} \sum_{l=1}^{m} f_{i}^{k, l} y_{i}^{k, l}
$$

(3) Objective 3: the lowest transportation risk for the decision of multimodal transportation scheme is as follows:

$$
\begin{aligned}
\operatorname{Min} R= & R T+\sum_{i=1}^{n} \sum_{k=1}^{m} x_{i, i+1}^{k} R C_{i, i+1}^{k} \\
& +\sum_{i=1}^{n} \sum_{k=1}^{m} \sum_{l=1}^{m} y_{i}^{k, l} R F_{i}^{k, l} .
\end{aligned}
$$

\subsection{Constraints of the Model}

(1) We can only choose one transportation mode between two nodes. Consider

$$
\sum_{k=1}^{m} x_{i, i+1}^{k}=1, \quad \forall i \in n .
$$

(2) Only transportation modes can be changed in a node. Consider

$$
\sum_{k=1}^{m} \sum_{l=1}^{m} y_{i}^{k, l}=1, \quad \forall i \in n
$$

(3) Ensure continuity during the transportation process. Consider

$$
x_{i-1, i}^{k}+x_{i, i+1}^{l} \geq 2 y_{i}^{k, l}, \quad \forall i \in n, \forall k \in m, \forall l \in m .
$$

(4) Goods transportation time should meet the actual time range allowed. Consider

$$
w \leq \sum_{i=1}^{n} \sum_{k=1}^{m} t_{i, i+1}^{k} x_{i, i+1}^{k}
$$

$$
+\sum_{i=1}^{n} \sum_{k=1}^{m} \sum_{l=1}^{m} f_{i}^{k, l} y_{i}^{k, l} \leq v, \quad v>w>0
$$

(5) Cargo weight should not exceed the transportation capacity. Consider

$$
Q x_{i, i+1}^{k} \leq A_{i, i+1}^{k}, \quad \forall i \in n, \forall k \in m, \forall l \in m .
$$

(6) The decision variable is 0 or 1 . Consider

$$
\begin{aligned}
& x_{i, i+1}^{k} \in\{0,1\}, \quad \forall i \in n, \forall k \in m, \forall l \in m, \\
& y_{i}^{k, l} \in\{0,1\}, \quad \forall i \in n, \forall k \in m, \forall l \in m .
\end{aligned}
$$

(7) Nonnegative constraints of variables. Consider

$$
\begin{aligned}
& t_{i, i+1}^{k} \geq 0, \quad \forall i \in n, \forall k \in m, \forall l \in m, \\
& f_{i}^{k, l} \geq 0, \quad \forall i \in n, \forall k \in m, \forall l \in m .
\end{aligned}
$$

2.6. Model Processing. Multiobjective programming model is difficult to solve because of the incoordination of the target vectors and the existence of constraints; in this paper, the objective function was simplified into a single objective function; usually we use the linear weighted method directly and sum multiple objective functions into a single objective function, but this method is only applicable to the transformation of the dimensionless objective function or uniform dimension objective function; it must be dimensionless or a dimensionally unified treatment for the multiobjective problem whose dimension is not uniform. We should change the original objective functions (2), (3), and (4) into the following:

$$
\begin{aligned}
\operatorname{Max} Z^{\prime}= & Z_{\max }-\sum_{i=1}^{n} \sum_{k=1}^{m} C_{i, i+1}^{k} X_{i, i+1}^{k} \\
& -\sum_{i=1}^{n} \sum_{k=1}^{m} \sum_{l=1}^{m} d_{i}^{k, l} y_{i}^{k, l}-J_{a}-S_{d}, \\
\operatorname{Max} T^{\prime}= & T_{\max }-\sum_{i=1}^{n} \sum_{k=1}^{m} t_{i, i+1}^{k} x_{i, i+1}^{k} \\
& -\sum_{i=1}^{n} \sum_{k=1}^{m} \sum_{l=1}^{m} f_{i}^{k, l} y_{i}^{k, l}, \\
\operatorname{Max} R^{\prime}= & R_{\max }-R T-\sum_{i=1}^{n} \sum_{k=1}^{m} x_{i, i+1}^{k} R C_{i, i+1}^{k} \\
& -\sum_{i=1}^{n} \sum_{k=1}^{m} \sum_{l=1}^{m} y_{i}^{k, l} R F_{i}^{k, l} .
\end{aligned}
$$


According to the evaluation function method of multiple objective function optimization method, referring to the weight degree of each target weight, we gave, respectively, the transportation cost, transportation time, and transportation risk weight as $\alpha, \beta$, and $\delta$ and $\alpha+\beta+\delta=1, \alpha, \beta, \delta \in[0,1]$.

At the same time, in order to solve the dimensional consistency problem of the objective function, we need to transform the target function (12):

$$
\begin{aligned}
\operatorname{Max} Z^{*}= & \left(Z_{\max }-\sum_{i=1}^{n} \sum_{k=1}^{m} C_{i, i+1}^{k} X_{i, i+1}^{k}\right. \\
& \left.-\sum_{i=1}^{n} \sum_{k=1}^{m} \sum_{l=1}^{m} d_{i}^{k, l} y_{i}^{k, l}-J_{a}-S_{d}\right) \\
& \times\left(Z_{\max }\right)^{-1}, \\
\operatorname{Max}^{*}= & \left(T_{\max }-\sum_{i=1}^{n} \sum_{k=1}^{m} t_{i, i+1}^{k} x_{i, i+1}^{k}\right. \\
& \left.\times\left(T_{\max }\right)^{-1}, \sum_{i=1}^{n} \sum_{k=1}^{m} \sum_{l=1}^{m} f_{i}^{k, l} y_{i}^{k, l}\right) \\
& \left(R_{\max }-R T-\sum_{i=1}^{n} \sum_{k=1}^{m} x_{i, i+1}^{k} R C_{i, i+1}^{k}\right. \\
\operatorname{Max}^{*}= & \left.-\sum_{i=1}^{n} \sum_{k=1}^{m} \sum_{l=1}^{m} y_{i}^{k, l} R F_{i}^{k, l}\right)
\end{aligned}
$$

In the objective function (13), $Z^{*}, T^{*}$, and $R^{*}$ are the goal function after dimensionless treatment, the range for the three is $[0,1)$. Among them, $Z_{\max }, T_{\max }$, and $R_{\max }$ are the respective maxima of target functions (2), (3), and (4) in each generation of the genetic algorithm; so, in this paper, we established the comprehensive objective function:

$$
f(Z, T, R)=\alpha \times Z^{*}+\beta \times T^{*}+\delta \times R^{*} .
$$

The states and effects of the three indexes weights in the comprehensive evaluation results are not the same in the comprehensive objective function (14); it is so important for the model solution when choosing the weight values. Generally speaking, in the decision of intermodal transportation scheme, the selected weight values mostly depend on the type of goods and the decision preference.

\section{Particle Swarm Optimization (PSO)}

3.1. Introduction of the Algorithm. Particle swarm optimization is an evolutionary computation technique, proposed in 1995 by Dr. Eberhart and Dr. Kennedy, from the behavior of birds' predation. The algorithm is initially rules by birds cluster activities of the enlightenment, using swarm intelligence to establish a simplified model. Particle swarm optimization algorithm is based on the observation of animal collective behavior; because of information sharing, the whole population movement from disorderly to orderly evolution process in the problem space by the individual in the group, then, obtained the optimal solution. PSO is similar to genetic algorithm; it is an optimization algorithm based on iteration. The system is initialized with a set of random solutions, searching for the optimal value through the iterative, but it has no genetic algorithm with the crossover and mutation; the particles search in the solution space to follow the optimal particle; PSO is simple and easy to realize and there is no need to adjust many parameters.

\subsection{The Advantages of PSO Algorithm}

(1) The algorithm is simple, with less adjustable parameters, and easy to implement.

(2) The random initialization population has strong global searching ability, which is similar to genetic algorithm.

(3) It uses the evaluation function to measure the individual searching speed.

(4) It has strong scalability.

\subsection{The Disadvantages of PSO Algorithm}

(1) The algorithm cannot make full use of the feedback information in the system.

(2) Its ability to solve combinatorial optimization problems is not strong.

(3) Its ability to solve the optimization problem is not very well.

(4) It is easy for this algorithm to obtain local optimal solution.

3.4. The Basic Principle of the Algorithm. The algorithm is described as follows: hypothesis in a $D$ dimension target searching space, $N$ particle to form a community, the $i$ particle vector to represent a $D$ dimension, and the position vector. Consider

$$
X_{i}=\left(x_{i 1}, x_{i 2}, \ldots, x_{i D}\right), \quad i=1,2, \ldots, N
$$

The velocity of vector $i$ particle is also a $D$ dimension, the velocity vector

$$
V_{i}=\left(v_{i 1}, v_{i 2}, \ldots, v_{i D}\right), \quad i=1,2, \ldots, N
$$


The $i$ particle is the searched best position called individual extreme value by far and is denoted as

$$
P_{\text {best }}=\left(p_{i 1}, p_{i 2}, \ldots, p_{i D}\right), \quad i=1,2, \ldots, N \text {. }
$$

The optimal position of the whole particle swarm searched so far is called global extreme and is denoted as

$$
g_{\text {best }}=\left(p_{g 1}, p_{g 2}, \ldots, p_{g D}\right)
$$

After finding out these two optimal values, particles updated their velocity and position according to the following formulas:

$$
\begin{gathered}
v_{i d}=w * v_{i d}+c_{1} r_{1}\left(p_{i d}-x_{i d}\right)+c_{2} r_{2}\left(p_{g d}-x_{i d}\right), \\
x_{i d}=x_{i d}+v_{i d} .
\end{gathered}
$$

Among them, $w$ is the inertia factor; $c_{1}$ and $c_{2}$ are the learning factors, and $r_{1}$ and $r_{2}$ are the ranges of $[0,1]$ for the uniform random numbers. The formula (19) is composed of three parts on the right: the first part reflects the movement of particles and represents particles which have maintained their previous velocity trend; the second part reflects memory particles on the historical experience and represents particles which are close to the optimal position of its historical trend; the third part reflects the cooperation and knowledge, sharing group history experience synergy between particles.

\section{Ant Colony Algorithm (ACO)}

4.1. Introduction of the Algorithm. Ant colony algorithm (ACO) is a new intelligent optimization algorithm proposed by Italian scholars M. Dorigo, V. Maniezzo, and A. Colorni in the twentieth century and early 90s; it has been applied to the TSP, secondary distribution and quadratic assignment problems, graph coloring problem, and many classical combination optimization problems and gained very good results.

\subsection{The Advantages of ACO Algorithm}

(1) It uses a kind of positive feedback mechanism and gets converges to the optimal path on the objective through the pheromone update ceaselessly.

(2) It is a kind of optimization method of distribution; it is easy to imply in parallel.

(3) It is a kind of method for global optimization, used not only for the single objective optimization problems, but also for solving the multiobjective optimization problems.

(4) It is suitable to solve the discretization problems.

(5) It has strong robustness.

\subsection{The Disadvantages of ACO Algorithm}

(1) The solving speed is slow because of the shortage in the initial stage of information algorithm.

(2) The algorithm based on the discrete problem cannot directly solve continuous optimization problems.

(3) This algorithm lacks strong searching ability.

4.4. The Basic Principle of the Algorithm. The basic principle of the algorithm is using $K$ ants jointly to construct a feasible solution; each ant is an independent one for the construction process of solution; the ants between each other exchange information through information value and constantly optimize the cooperative solution and at the same time give the ants distribution list (recorded the visited point of this ant); introduce the other one ant to follow the first ant's behavior; continue to search in the remaining points. The ant's task is under many constraints; look for communicating nodes one by one, and leave the pheromone in the connected node arc. Use arc pheromone and heuristic information to determine assignment order and use local optimization of pheromone to update; after all, the $k$ ants are constructed completing the application, then apply to the global pheromone update.

$\tau_{i j}(t)$ stated at the $t$ moment in the node $i, j$ connection information; $\alpha$ is the importance degree of residual information in ant path $i, j ; \eta_{i j}$ is the heuristic information, the expected degree of ants from node $i$ to node $j$; in this paper, $\eta_{i j}=1 / d_{i j}\left(d_{i j}\right.$ is the distance between node $i$ and node $j$ ); $\beta$ is the importance degree of the heuristic information. The initial time of nodes, each ant was placed on the random selection path construction according to the transition probabilities; the $K$ ant's transfer probability of node $i$ to node $j$ at $t$ time is $P_{i j}^{k}$, which is feasible in the ant $K$ sentinel node $i$ set. Consider

$$
p_{i j}^{k}(t)= \begin{cases}0, & \text { otherwise, } \\ \frac{\left[\tau_{i j}(t)\right]^{\alpha}\left[\eta_{i j}\right]^{\beta}}{\sum_{i \in N_{i}^{k}}\left[\tau_{i j}(t)\right]^{\alpha}\left[\eta_{i j}\right]^{\beta}}, & j \in N_{i}^{k} .\end{cases}
$$

According to the rules and update time determined by different update quantity of information, all the ants of each group after the completion of travel in the set the pheromone should be adjusted; the ant density model was used in this paper; that is to say, ants moved from node $i$ to node $j$ and updated pheromone of the path $i j$. Among them, $\Delta \tau_{i j}^{k}$ shows the number of unit length of messages of ant $K$ in the path from $i$ to $j ; \gamma$ is evaporation coefficient of pheromone; constant $Q$ is ants leaving tracks number. Consider

$$
\Delta \tau_{i j}^{k}(t, t+1)= \begin{cases}Q, & \text { ant } k \text { transfers from node } i \text { to } \\ & \text { node } j \text { at the } t \text { time to } t+1 \text { time } \\ 0, & \text { otherwise. }\end{cases}
$$


Update rules corresponding information is as follows:

$$
\begin{aligned}
\tau_{i j}(t+1) & =(1-\gamma) \tau_{i j}(t)+\Delta \tau_{i j}(t, t+1), \\
\Delta \tau_{i j}(t, t+1) & =\sum_{k=1}^{m} \Delta \tau_{i j}^{k}(t, t+1) .
\end{aligned}
$$

\section{Particle Swarm and Ant Colony Algorithm (PSACO)}

5.1. Introduction of the Algorithm. In this paper, PSACO algorithm which combines the particle swarm algorithm and ant colony algorithm is proposed, combining the advantages of two kinds of algorithms and make up for the disadvantages of the two algorithms. Particle swarm algorithm is more suitable for solving continuous optimization problems and is slightly inferior in solving combinatorial optimization problems, but, due to the random distribution of the initial particle, we use it to solve the combinatorial optimization problems; the algorithm has better global search ability and fast solution speed; the ant colony algorithm is better in solving combinatorial optimization problems than the particle swarm optimization algorithm, because the initial distribution is uniform, which makes the ant colony algorithm with blindness at the beginning of the algorithm, so it does not converge very quickly. PSACO algorithm combines particle swarm algorithm and ant colony algorithm, absorbs the advantages of the two algorithms, and overcomes the shortcomings; the advantage is complementary and better than that of ant colony algorithm in time efficiency and the particle swarm algorithm in accuracy and efficiency.

5.2. The Basic Ideas and Steps of PSACO Algorithm. The PSACO algorithm combines advantages of particle swarm algorithm and ant colony algorithm, and its basic idea is as follows: in the first stage, the PSACO algorithm makes full use of random, rapid, global features of the particle swarm optimization algorithm, and, after a certain number of iterations, finds out the suboptimal solution of the problem and adjusts the initial distribution; in the second stage of the algorithm, PSACO algorithm uses information distribution in the first stage, makes full use of parallelism, positive feedback, and high accuracy advantages of ant colony algorithm, and completes the entire problem solving progress. Specifically, according to the characteristics of the solution problem, combining two groups of algorithms is effective to solve the combinatorial optimization problem of multimodal transportation choice mode.

The PSACO algorithm is divided into two parts; firstly, particle swarm algorithm generates transportation connectivity nodes from node start to node finish and then uses the ant colony algorithm to find the optimal combination of the sequence nodes of the transportation mode; the basic steps are as follows.

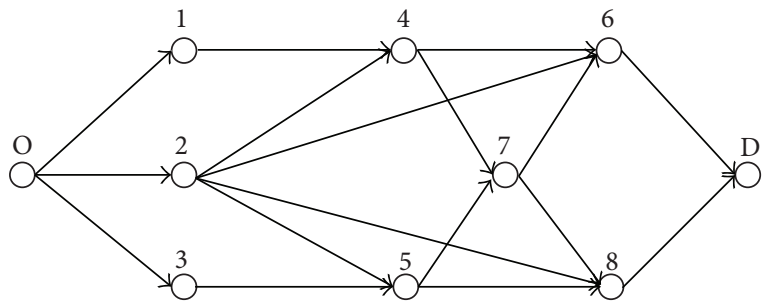

FIGURE 1: Schematic diagram of a multimodal transportation network.

Step 1. Particle swarm algorithm parameters and variables are initialized.

Step 2. Set the ant colony algorithm parameters initialization and each ant's position as follows:

(1) according to the selection probability formula (21) and the roulette wheel method, choose the path for each ant;

(2) when structural path, each ant updates pheromone locally at the same time according to the formula (23);

(3) execute (1), (2), and (3) circularly until every ant generates a complete path;

(4) record and calculate the objective function value of each ant according to the tabu list and give the optimal and worst ant;

(5) update global pheromone for the best ant and worst ant according to formula (23);

(6) execute (2)-(6) until they meet the end condition.

Step 3. Call the ant colony algorithm to get each particle's fitness and update the historical optimum particle position vector and global optimum particle position vector.

Step 4. Update the particle velocity vector and the position vector according to the formula (19) and (20).

Step 5. Execute Steps 2-4 to satisfy the end conditions and use the ant colony algorithm to get the optimal solution.

Step 6. End.

\section{Example}

For example, a transportation enterprise needs to transport 100 tons of goods from city $O$ to city $D$; each passing section has four kinds of transportation modes to select. Figure 1 shows the schematic diagram of a multimodal transportation network. Table 1 shows the transportation modes that we can choose between two adjacent city nodes, the transportation costs, transportation time, and risk values about different transportation modes. Table 2 shows the unit transfer cost, unit transit time, and transfer risk about different transportation modes. The cargo delivery time interval was [ $130 \mathrm{~h}, 165 \mathrm{~h}$ ] 
TABLE 1: Transportation costs/transportation time/transportation risk/transportation capacity of different transportation modes.

\begin{tabular}{|c|c|c|c|c|c|c|}
\hline Start node & Terminal node & $\begin{array}{c}\text { Transportation } \\
\text { mode }\end{array}$ & $\begin{array}{c}\text { Transportation } \\
\text { costs/yuan }\end{array}$ & $\begin{array}{c}\text { Transportation } \\
\text { time/hour }\end{array}$ & Transportation risk & $\begin{array}{c}\text { Transportation } \\
\text { capacity }\end{array}$ \\
\hline $\mathrm{O}$ & 1 & Railway & 35 & 1 & 1 & 120 \\
\hline $\mathrm{O}$ & 1 & Road & 20 & 2 & 3 & 115 \\
\hline $\mathrm{O}$ & 1 & Waterway & 18 & 1.5 & 1 & 90 \\
\hline $\mathrm{O}$ & 1 & Aviation & 40 & 0.5 & 2 & 120 \\
\hline $\mathrm{O}$ & 2 & Railway & 25 & 0.8 & 1 & 100 \\
\hline $\mathrm{O}$ & 2 & Road & 15 & 1.5 & 2 & 115 \\
\hline $\mathrm{O}$ & 2 & Waterway & 13 & 1 & 1 & 120 \\
\hline $\mathrm{O}$ & 2 & Aviation & 35 & 0.3 & 2 & 125 \\
\hline $\mathrm{O}$ & 3 & Railway & 30 & 1.2 & 1 & 120 \\
\hline $\mathrm{O}$ & 3 & Road & 18 & 2.2 & 2.5 & 95 \\
\hline $\mathrm{O}$ & 3 & Waterway & 13 & 1.8 & 1.2 & 110 \\
\hline $\mathrm{O}$ & 3 & Aviation & 36 & 0.5 & 1.8 & 100 \\
\hline 1 & 4 & Railway & 45 & 1.5 & 1 & 90 \\
\hline 1 & 4 & Road & 25 & 2.5 & 3 & 110 \\
\hline 1 & 4 & Waterway & 20 & 2 & 1 & 120 \\
\hline 1 & 4 & Aviation & 42 & 0.7 & 2 & 110 \\
\hline 2 & 4 & Railway & 50 & 2 & 2 & 100 \\
\hline 2 & 4 & Road & 30 & 3 & 4 & 105 \\
\hline 2 & 4 & Waterway & 30 & 2.5 & 2 & 105 \\
\hline 2 & 4 & Aviation & 44 & 0.9 & 2 & 90 \\
\hline 2 & 5 & Railway & 55 & 2.5 & 2 & 100 \\
\hline 2 & 5 & Road & 35 & 3.5 & 4 & 100 \\
\hline 2 & 5 & Waterway & 32 & 2.8 & 2 & 130 \\
\hline 2 & 5 & Aviation & 46 & 1.2 & 2 & 95 \\
\hline 2 & 6 & Railway & 65 & 3.5 & 3 & 95 \\
\hline 2 & 6 & Road & 45 & 4.5 & 4.5 & 100 \\
\hline 2 & 6 & Waterway & 33 & 3.5 & 3 & 110 \\
\hline 2 & 6 & Aviation & 50 & 1.8 & 2.5 & 110 \\
\hline 2 & 8 & Railway & 70 & 4 & 4 & 110 \\
\hline 2 & 8 & Road & 50 & 5 & 5 & 100 \\
\hline 2 & 8 & Waterway & 58 & 4 & 3 & 150 \\
\hline 2 & 8 & Aviation & 55 & 2 & 2.5 & 100 \\
\hline 3 & 5 & Railway & 43 & 1.4 & 1 & 100 \\
\hline 3 & 5 & Road & 22 & 2.3 & 2.5 & 110 \\
\hline 3 & 5 & Waterway & 20 & 2 & 1 & 120 \\
\hline 3 & 5 & Aviation & 40 & 0.8 & 1.8 & 110 \\
\hline 4 & 6 & Railway & 24 & 1 & 1 & 100 \\
\hline 4 & 6 & Road & 16 & 1.5 & 2 & 115 \\
\hline 4 & 6 & Waterway & 24 & 1 & 1 & 120 \\
\hline 4 & 6 & Aviation & 30 & 0.3 & 2 & 125 \\
\hline 4 & 7 & Railway & 35 & 2 & 3 & 105 \\
\hline 4 & 7 & Road & 25 & 3 & 4 & 95 \\
\hline 4 & 7 & Waterway & 20 & 2 & 2 & 120 \\
\hline 4 & 7 & Aviation & 38 & 1.5 & 2.5 & 100 \\
\hline 5 & 7 & Railway & 35 & 2 & 3.5 & 100 \\
\hline 5 & 7 & Road & 24 & 2.5 & 3.5 & 100 \\
\hline 5 & 7 & Waterway & 30 & 1.8 & 2 & 115 \\
\hline
\end{tabular}


TABLE 1: Continued.

\begin{tabular}{|c|c|c|c|c|c|c|}
\hline Start node & Terminal node & $\begin{array}{c}\text { Transportation } \\
\text { mode }\end{array}$ & $\begin{array}{c}\text { Transportation } \\
\text { costs/yuan }\end{array}$ & $\begin{array}{c}\text { Transportation } \\
\text { time/hour }\end{array}$ & Transportation risk & $\begin{array}{c}\text { Transportation } \\
\text { capacity }\end{array}$ \\
\hline 5 & 7 & Aviation & 36 & 1.5 & 2.5 & 100 \\
\hline 5 & 8 & Railway & 35 & 1 & 1 & 120 \\
\hline 5 & 8 & Road & 20 & 2 & 3 & 115 \\
\hline 5 & 8 & Waterway & 32 & 1.5 & 1 & 90 \\
\hline 5 & 8 & Aviation & 40 & 0.5 & 2 & 120 \\
\hline 6 & $\mathrm{D}$ & Railway & 30 & 1.5 & 2 & 100 \\
\hline 6 & $\mathrm{D}$ & Road & 20 & 2 & 3 & 95 \\
\hline 6 & $\mathrm{D}$ & Waterway & 18 & 1.5 & 2 & 120 \\
\hline 6 & $\mathrm{D}$ & Aviation & 35 & 1 & 2 & 125 \\
\hline 7 & 6 & Railway & 40 & 1.5 & 2 & 100 \\
\hline 7 & 6 & Road & 20 & 2.5 & 2.5 & 110 \\
\hline 7 & 6 & Waterway & 24 & 2 & 1 & 120 \\
\hline 7 & 6 & Aviation & 43 & 1 & 2 & 110 \\
\hline 7 & 8 & Railway & 42 & 2 & 3 & 100 \\
\hline 7 & 8 & Road & 20 & 2.5 & 3 & 110 \\
\hline 7 & 8 & Waterway & 25 & 2.5 & 2 & 120 \\
\hline 7 & 8 & Aviation & 45 & 1.5 & 4 & 110 \\
\hline 8 & $\mathrm{D}$ & Railway & 45 & 1.5 & 1 & 90 \\
\hline 8 & $\mathrm{D}$ & Road & 25 & 2.5 & 3 & 110 \\
\hline 8 & $\mathrm{D}$ & Waterway & 20 & 2 & 1 & 120 \\
\hline 8 & $\mathrm{D}$ & Aviation & 42 & 0.7 & 2 & 110 \\
\hline
\end{tabular}

TABLE 2: Unit transfer costs (yuan)/unit transit time (second)/transfer risk of different transportation modes.

\begin{tabular}{lcccc}
\hline & Railway & Road & Waterway & Aviation \\
\hline Railway & $0 / 0 / 0$ & $2 / 2 / 2$ & $3 / 4 / 3$ & $4 / 4 / 3$ \\
Road & $2 / 2 / 2$ & $0 / 0 / 0$ & $3 / 4 / 4$ & $4 / 3 / 4$ \\
Waterway & $3 / 4 / 3$ & $3 / 4 / 4$ & $0 / 0 / 0$ & $4 / 3 / 3$ \\
Aviation & $4 / 4 / 3$ & $4 / 3 / 4$ & $4 / 3 / 3$ & $0 / 0 / 0$ \\
\hline
\end{tabular}

in the contract; the goods' penalty cost coefficient was 6 yuan/hour, when delivery advanced, and 10 yuan/hour, when delivery is late, the risk factor to send the goods was 3 . How to choose the multimodal transportation path and let the transportation enterprise find the optimal scheme to save transportation costs, reduce the total transportation time, and reduce the total risk.

According to the example, by combining decision makers' multimodal transportation needs, the weights of transportation costs, transportation time, and transportation risk value were set to $0.6,0.1$, and 0.3 (different decisions will lead to different weights setting); this paper adopted MATLAB to verify the effectiveness of the proposed algorithm; first the 20 iterations of particle swarm algorithm were the initial solution of the problem; then we used the results of the initial solution by ant colony algorithm for distribution. $\gamma=0.02$ in ant colony algorithm; the number of ants was equal to the multimodal transportation city node number; $\alpha=2$ and $\beta=5$;
Table 3 shows the comparison of PSACO algorithm and basic ant colony algorithm in solving ability and efficiency.

It can be seen from the simulation results that the PSACO algorithm is compared with the basic ACO algorithm; it has not only better results than the ant colony algorithm, but also higher computation efficiency; that is, because, after the particle swarm algorithm, initial pheromone distribution was improved, avoiding the basic ACO algorithm for blind initial pheromone distribution searching; thus, it is helpful for ant colony algorithm to get more accurate searches. Table 4 shows the optimal transport scheme.

\section{Conclusion}

This paper is about the decision problem of multimodal transportation scheme; it searched and analyzed relevant research literatures at home and abroad, combined them with the diverse needs of transportation scheme decision, and proposed and established combinatorial optimization mathematical model of intermodal transportation plan decision based on transportation time and transportation cost and risk; at the same time, it used heuristic algorithm which combined particle swarm optimization algorithm and ant colony algorithm to solve the model and got the transportation scheme optimization. The experiments showed that the algorithm has achieved very good results in time performance and optimization performance; it was a feasible algorithm. The content of this paper enriched and perfected 
TABLE 3: The results and comparison of PSACO algorithm.

\begin{tabular}{lcccc}
\hline Algorithm & $\begin{array}{c}\text { The comprehensive } \\
\text { evaluation value }\end{array}$ & $\begin{array}{c}\text { Transportation } \\
\text { time/hour }\end{array}$ & $\begin{array}{c}\text { Transportation } \\
\text { costs/yuan }\end{array}$ & $\begin{array}{c}\text { Transportation } \\
\text { risks }\end{array}$ \\
\hline PSACO algorithm & 0.438 & 150.6 & 4258 & 6.7 \\
ACO algorithm & 0.486 & 156.8 & 4456 & 28 \\
\hline
\end{tabular}

TABLE 4: The optimal transport scheme.

\begin{tabular}{lc}
\hline \multicolumn{2}{c}{ The optimal transport scheme } \\
\hline $\begin{array}{l}\text { Transportation route } \\
\text { O-2-4-7-8-D }\end{array}$ \\
Transportation mode & Railway-road-waterway-railway-aviation \\
\hline
\end{tabular}

the theories and methods of the hot issues in the study of intermodal transportation scheme decision, and the results provided the references and reasonable arrangements for the transportation process and transportation project decision making.

\section{Conflict of Interests}

The authors declare that there is no conflict of interests regarding the publication of this paper.

\section{References}

[1] K. Chen and L. Ma, "Glowworm swarm optimization algorithm for 0-1 knapsack problem," Application Research of Computers, vol. 4, no. 30, pp. 993-995, 2013.

[2] B. Li, X. Q. Yan, and J. X. Hu, "Model of container terminal logistics optimization system based on the Harvard structure and swarm intelligence," Journal of Jiangsu University of Science and Technology, vol. 3, no. 25, pp. 282-286, 2011.

[3] J. Mi, "Improved swarm intelligence optimization algorithm used in the task sorting," Journal of Beijing Information Science and Technology University, vol. 3, no. 25, pp. 13-18, 2010.

[4] H. Li, X. Chen, and M. Zhang, "The path planning problem based on swarm intelligence algorithm," Journal of Tsinghua University, vol. 3, pp. 1770-1773, 2007.

[5] B. Wu and Z. Z. Shi, "A kind of TSP problem based on ant colony algorithm partition algorithm," Journal of Computers, vol. 12, pp. 1328-1333, 2001.

[6] R.-B. Xiao, Z.-W. Tao, and H.-F. Zou, "Concurrent tolerance optimization design based on hybrid swarm intelligence algorithm," Computer Integrated Manufacturing Systems, vol. 13, no. 4, pp. 668-674, 2007.

[7] Q. L. Ma, W. N. Liu, D. H. Sun, and Y. F. Dan, "Travel route planning model based on fusion of multi intelligent algorithm," Computer Science, vol. 10, pp. 211-221, 2010.

[8] A. Lozano and G. Storchi, "Shortest viable path algorithm in multimodal networks," Transportation Research A: Policy and Practice, vol. 35, no. 3, pp. 225-241, 2001.

[9] H. I. Calvete, C. Galé, M.-J. Oliveros, and B. Sánchez-Valverde, "A goal programming approach to vehicle routing problems with soft time windows," European Journal of Operational Research, vol. 177, no. 3, pp. 1720-1733, 2007.
[10] Y. Li and J. Zhao, "Choice of multimodal transportation modal with fixed freight," Journal of Southwest Jiaotong University, vol. 47, no. 5, pp. 881-887, 2012.

[11] X. Wang, Z. B. Chi, and X. L. Ge, "Whole vehicle multimodal transportation model research and analysis with time windows," Computer Research and Application, vol. 28, no. 2, pp. 563-565, 2011.

[12] F. L. Feng and Q. Y. Zhang, “Organization collaborative optimization model of multimodal transportation based on environment," Journal of Railway Science and Engineering, vol. 9, no. 6, pp. 95-100, 2012.

[13] L. Tong and L. Nie, "Multimodal transportation path optimization model and method research," Logistics Technology, vol. 29, no. 5, pp. 57-60, 2010.

[14] J. W. Zhang, "Multimodal container transportation network path rationalization process," Journal of Beijing Jiaotong University, 2011.

[15] L. Li and Y. C. Zeng, "Multimodal transportation model and algorithm in logistics transportation," Statistics and Decision Making, vol. 20, pp. 27-29, 2009.

[16] J. Wen, "Optimization model to control the cost of container multimodal transportation," Changsha Science and Technology University, 2010.

[17] L. Li, "Multimodal transportation modes generalized cost optimal choice under conditions," Journal of Beijing Jiaotong University, 2006.

[18] X. X. Wang, "The multimodal transportation minimum cost problem with time window," Beijing Posts and Telecommunications University, 2009.

[19] J. Zhang, "Railway container transportation network model and algorithm," Central South University, 2007.

[20] Z. J. Jia, "The risks analysis and control of the goods multimodal transportation," Chongqing Jiaotong University, 2009.

[21] F. Liu, "The container multimodal transportation corridor planning and transportation modes selection," Journal of Southwest Jiaotong University, 2011.

[22] X. K. Luo, "Optimization algorithm research of logistics and multimodal transportation," Shanghai Jiaotong University, 2004. 


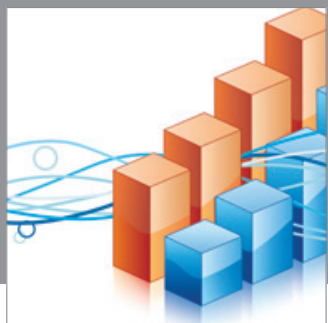

Advances in

Operations Research

mansans

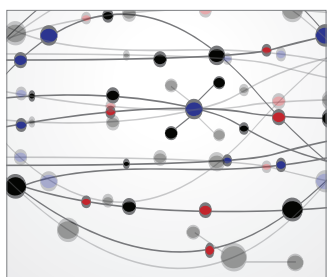

The Scientific World Journal
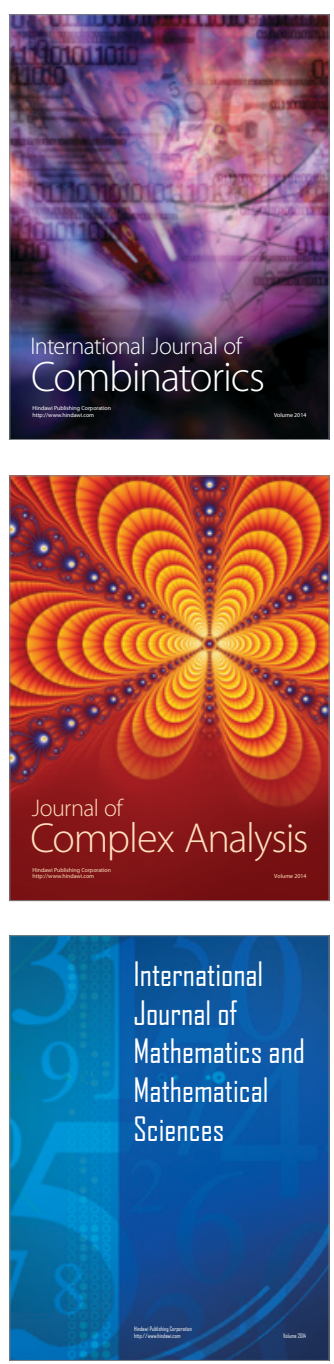
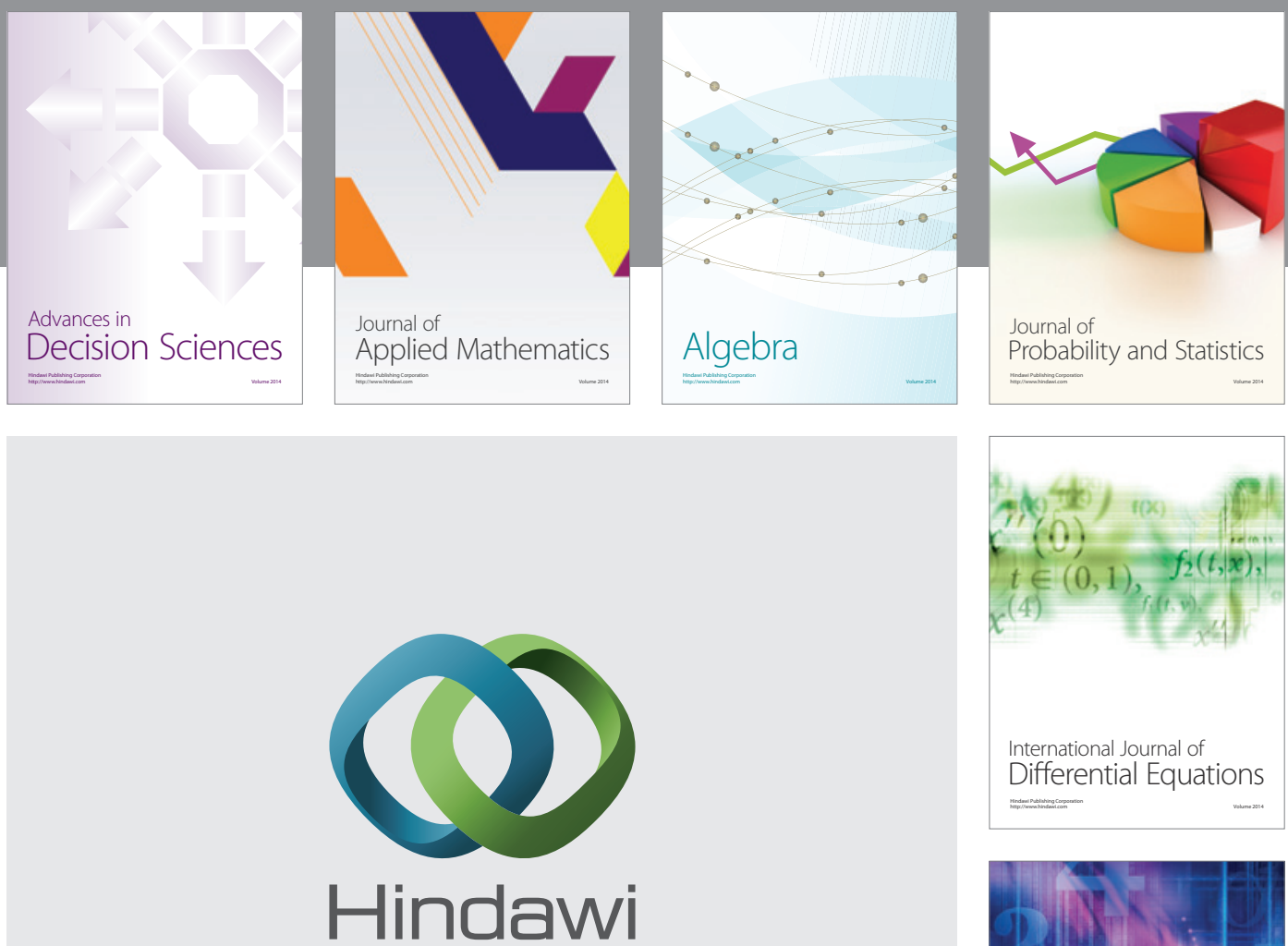

Submit your manuscripts at http://www.hindawi.com
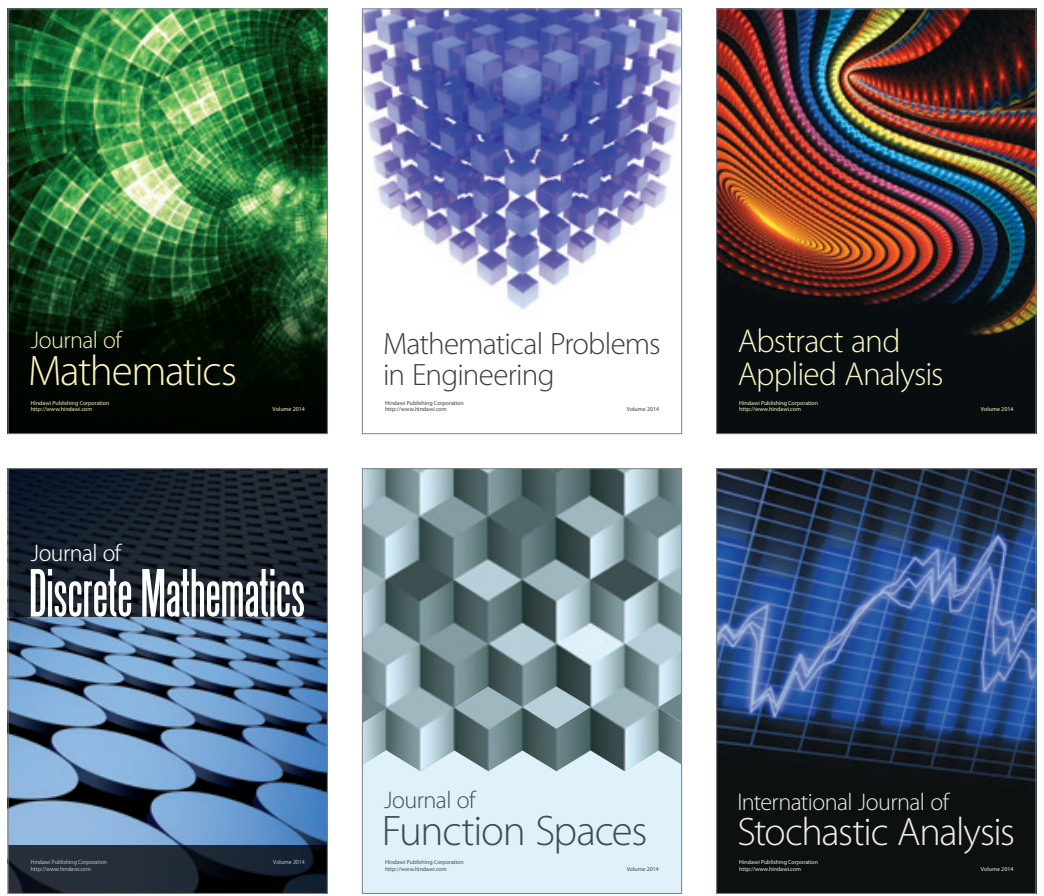

Journal of

Function Spaces

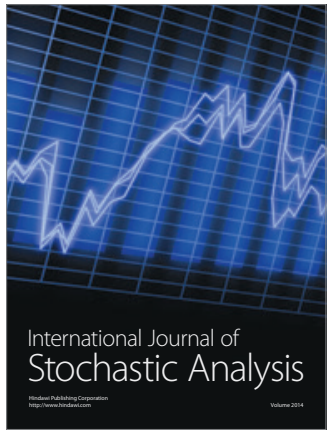

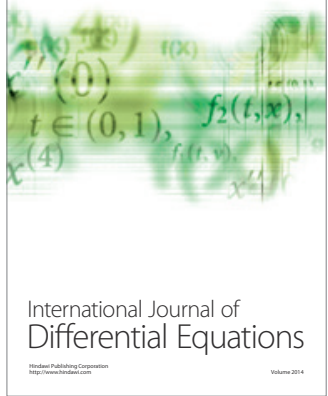
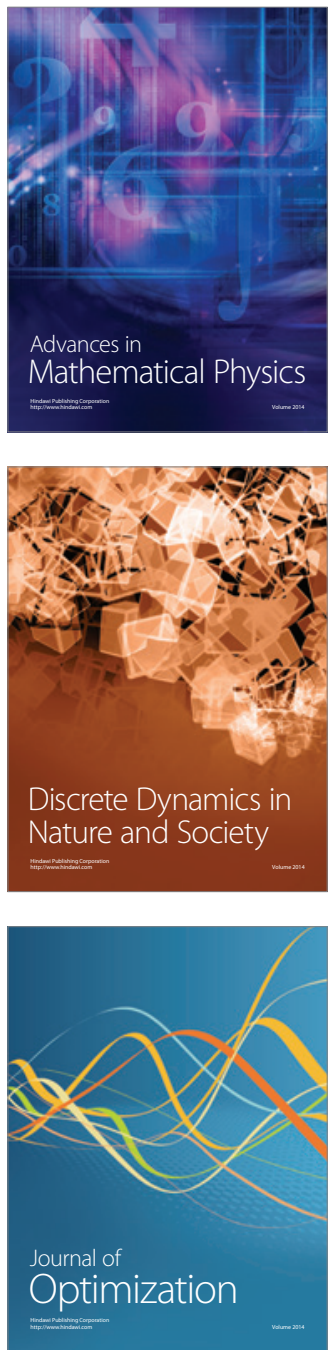